\title{
Produção de geopolímeros utilizando cinza da casca de arroz como fonte complementar de sílica
}

\section{(Production of geopolymers using rice husk ash as supplementary source of silica)}

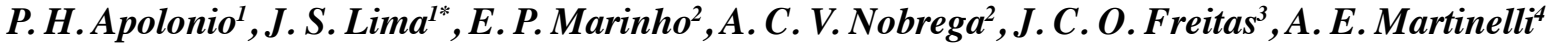 \\ ${ }^{1}$ Universidade Federal de Pernambuco, Programa de Pós-graduação em Engenharia Civil, \\ Centro de Tecnologia e Geociências, Recife, PE, Brasil \\ ${ }^{2}$ Universidade Federal de Pernambuco, Programa de Pós-graduação em Engenharia Civil e Ambiental, \\ Caruaru, PE, Brasil \\ ${ }^{3}$ Universidade Federal do Rio Grande do Norte, Departamento de Química, Natal, RN, Brasil \\ ${ }^{4}$ Universidade Federal do Rio Grande do Norte, Programa de Pós-graduação em Ciência e \\ Engenharia de Materiais, Natal, RN, Brasil
}

\begin{abstract}
Resumo
O silicato de sódio é a fonte complementar utilizada na reação de geopolimerização. Entretanto, esse reagente é produzido em alta temperatura ou em autoclave a alta pressão, o que gera muito impacto ambiental. Visando a produção de geopolímeros mais ecológicos, produziu-se um silicato de sódio alternativo (ASW) a partir da dissolução da sílica presente na cinza de casca de arroz (CCA). Foram preparados geopolímeros nas razões molares $\mathrm{SiO}_{2} / \mathrm{Al}_{2} \mathrm{O}_{3}$ de 3,0, 3,5 e 4,0 e com concentrações de $\mathrm{NaOH}$ de 8 e $10 \mathrm{M}$. Os corpos de prova foram submetidos a ensaios de resistência à compressão (7 e 28 dias), FTIR e DRX. O desempenho mecânico dos geopolímeros produzidos com ASW foi semelhante aos produzidos com silicato de sódio comercial. A análise do comportamento mecânico e dos espectros de infravermelho sugeriu que ocorreu maior dissolução da CCA com o aumento da relação molar $\mathrm{SiO}_{2} / \mathrm{Al}_{2} \mathrm{O}_{3}$ e da concentração do álcali.

Palavras-chave: geopolímero, cinza da casca de arroz, silicato de sódio, fonte alcalina.
\end{abstract}

\begin{abstract}
Sodium silicate is a complementary source used in the geopolymerization reaction. However, this reagent is produced at high temperature or in an autoclave at high pressure, which generates a significant environmental impact. Aiming the production of greener geopolymers, an alternative sodium silicate (ASW) from the dissolution of silica present in rice husk ash (RHA) was produced. Geopolymers were prepared at $\mathrm{SiO}_{2} / \mathrm{Al}_{2} \mathrm{O}_{3}$ molar ratios of 3.0, 3.5, and 4.0, and concentrations of 8 and $10 \mathrm{M} \mathrm{NaOH}$. The specimens were subjected to compressive strength ( 7 and 28 days), FTIR and XRD tests. The mechanical performance of ASWproduced geopolymers was similar to those made with commercial sodium silicate. Analysis of the mechanical behavior and infrared spectra suggested that there was a greater dissolution of $\mathrm{RHA}$ with increasing $\mathrm{SiO}_{2} / \mathrm{Al}_{2} \mathrm{O}_{3}$ molar ratio and alkali concentration.

Keywords: geopolymer, rice husk ash, sodium silicate, alkaline source.
\end{abstract}

\section{INTRODUÇÃO}

Os geopolímeros são polímeros inorgânicos reticulados de longa cadeia formados por unidades tetraédricas de $\mathrm{AlO}_{4}$ e $\mathrm{SiO}_{4}$, obtidos em meio fortemente alcalino [1,2]. Possuem uma estrutura tridimensional no estado amorfo [1], resultante da polimerização de monômeros de aluminossilicato em uma solução alcalina [3]. Os geopolímeros apresentam alta resistência à compressão e durabilidade [1, 4-6], baixa retração, baixa condutividade térmica, resistência ao fogo, resistência ao ácido $[4,5,7]$, resistência à corrosão

*jofresl@gmail.com

Dhttps://orcid.org/0000-0001-6097-6230 e resistência à umidade [8]. Entre as diversas aplicações dos materiais geopoliméricos, destaca-se seu uso como aglomerante. Entre os componentes que são utilizados para a produção do concreto geopolimérico, o mais impactante do ponto de vista energético e da emissão de $\mathrm{CO}_{2}$ é o silicato de sódio que demanda $63,4 \%$ da energia e é responsável por $67,2 \%$ da emissão de $\mathrm{CO}_{2}$ [9]. Os silicatos de sódio e potássio comerciais são utilizados como fonte complementar de sílica (solúvel na solução ativadora) na produção do geopolímero, e leva à formação de uma estrutura compacta e densa, com alta resistência mecânica. Entretanto, este componente é o mais caro para produção do geopolímero e sua produção provoca grande impacto ambiental, devido ao seu processo de fabricação $[10,11]$. 
Sabe-seque aemissão de $\mathrm{CO}_{2}$ peloconcreto geopolimérico é até 3,6 vezes menor do que a emissão pelo concreto tradicional. A síntese de geopolímeros pode ser ainda mais ecológica se forem utilizadas matérias-primas mais limpas para produção de silicato de sódio. Ativadores alcalinos baseados em silicato de potássio comercial fornecem uma emissão maior de $\mathrm{CO}_{2}$, aproximadamente $1,1 \mathrm{ton} / \mathrm{m}^{3}$ de geopolímero produzido, enquanto ativadores baseados em fontes alternativas geram em torno de $572,3 \mathrm{~kg} / \mathrm{m}^{3}$ para a cinza da casca de arroz e $660,2 \mathrm{~kg} / \mathrm{m}^{3}$ para a sílica ativa, sendo assim, considerados materiais mais ecologicamente corretos [12]. Em todo o mundo e no Brasil a produção de arroz é grande, atingindo em 2014 cerca de 741,3 milhões [13] e 12,1 milhões [13] de toneladas, respectivamente. A casca de arroz quando queimada nas caldeiras gera cerca de $25 \%$ de cinza, o que corresponde a aproximadamente 40,8 milhões de toneladas/ano de cinza da casca de arroz no mundo, sendo 665,5 mil toneladas no Brasil [14]. A cinza da casca de arroz (CCA) torna-se uma opção interessante para produção do silicato de sódio por possuir alto teor de $\mathrm{SiO}_{2}$ amorfo (80-95\% em massa) [15], podendo ser dissolvida em solução alcalina [2, 6, 16-20]. Além disso, a casca do arroz é o principal resíduo vegetal na produção de cinza através do processo de incineração [21].

Diversos estudos [2, 6, 16-20] apontam o silicato de sódio obtido através de CCA como um ativador alcalino eficiente para produção de geopolímeros, apresentando valores de resistência mecânica semelhante aos valores obtidos com silicatos de sódio comerciais. No entanto, faltam estudos mais aprofundados referentes à substituição total do silicato de sódio comercial pelo silicato de sódio produzido através da dissolução de CCA em hidróxido de sódio para produção de geopolímeros. O objetivo desta pesquisa foi sintetizar silicato de sódio a partir da dissolução em meio alcalino da sílica existente na CCA e uso deste silicato como fonte complementar para produção de geopolímeros. Para tal, foi estudada a influência da concentração do hidróxido de sódio e da relação $\mathrm{SiO}_{2} / \mathrm{Al}_{2} \mathrm{O}_{3}$ na resistência à compressão e na estrutura, por espectroscopia no infravermelho com transformada de Fourier (FTIR) e difração de raios X (DRX), das pastas geopoliméricas produzidas.

\section{MATERIAIS E MÉTODOS}

Materiais: o precursor geopolimérico empregado para produção dos geopolímeros foi o Metacaulim HP (MK) da Metacaulim do Brasil. Foi utilizado hidróxido de sódio (pureza mínima de 97\%) da Química Moderna e o silicato de sódio neutro R3342 da Diatom Mineração como fonte complementar de sílica. A cinza de casca de arroz (CCA) utilizada foi Silroz 325 da Marina Tecnologia (Rio Grande Sul/Brasil). As características físico-químicas das matériasprimas são apresentada na Tabela I. O pó de CCA se apresentou como uma estrutura composta principalmente de partículas irregulares e porosas [22].

Síntese do silicato de sódio: foram produzidas soluções ativadoras usando CCA como fonte de sílica. Os silicatos de
Tabela I - Características físico-químicas do metacaulim (MK), silicato de sódio comercial (SW) e cinza de casca de arroz (CCA).

[Table I - Physico-chemical characteristics of metakaolin $(M K)$, commercial sodium silicate (SW), and rice husk ash (RHA).]

\begin{tabular}{|c|c|c|c|}
\hline Característica & MK & $\mathrm{SW}^{\#}$ & CCA \\
\hline $\mathrm{SiO}_{2}(\%)$ & 53,06 & 30,0 & 80,00 \\
\hline $\mathrm{Al}_{2} \mathrm{O}_{3}(\%)$ & 36,50 & - & - \\
\hline $\mathrm{Na}_{2} \mathrm{O}(\%)$ & - & 9,0 & - \\
\hline $\mathrm{K}_{2} \mathrm{O}(\%)$ & 1,36 & - & 3,44 \\
\hline $\mathrm{CaO}(\%)$ & 0,86 & - & 1,14 \\
\hline $\mathrm{MgO}(\%)$ & 0,27 & - & - \\
\hline $\mathrm{Fe}_{2} \mathrm{O}_{3}(\%)$ & 4,42 & - & 0,82 \\
\hline $\mathrm{MnO}(\%)$ & - & - & 2,85 \\
\hline $\mathrm{SO}_{3}(\%)$ & - & - & 1,34 \\
\hline Outros $(\%)$ & 1,37 & - & 0,82 \\
\hline Perda ao fogo $(\%)$ & 2,15 & 39,0 & 9,59 \\
\hline Umidade $(\%)$ & 1,18 & 61,0 & 2,41 \\
\hline Massa específica $\left(\mathrm{g} / \mathrm{cm}^{3}\right)$ & 2,53 & 1,41 & 2,16 \\
\hline Área específica* $\left(\mathrm{m}^{2} / \mathrm{kg}\right)$ & 1242 & - & 1672 \\
\hline
\end{tabular}

sódio foram preparados em solução aquosa de $\mathrm{NaOH}$ (8 e 10 M). As soluções foram aquecidas em sistema de refluxo a $90^{\circ} \mathrm{C}$ por $2 \mathrm{~h}$. Para garantir a temperatura e uniformidade do sistema, foi utilizado evaporador rotativo, mantendo rotação controlada em 2 rpm em banho de óleo.

Síntese do geopolímero: os geopolímeros foram preparados adicionando a solução ativadora gradativamente ao metacaulim e misturadodurante $5 \mathrm{~min}$.Um resumo das misturas está relatado na Tabela II. Após mistura, as amostras de geopolímero fresco foram colocadas rapidamente em recipientes cilíndricos com dimensões 25x50 mm confeccionados em PVC [23]. A cura das amostras de geopolímero foi realizada ao ar e temperatura ambiente $\left(25^{\circ} \mathrm{C}\right)$.

Caracterização das matérias-primas, silicatos de sódio e geopolímeros: as composições químicas do metacaulim (MK) e da cinza de casca de arroz (CCA) foram determinadas por fluorescência de raios X (XRF), em espectrômetro Ray Ny EDX-720, Shimadzu. As análises de DRX foram feitas no difratômetro XRD-6000, Shimadzu, com as seguintes condições de operação: radiação $\mathrm{CuK} \alpha(40 \mathrm{kV} / 30 \mathrm{~mA})$, velocidade do goniômetro de $0,02^{\circ} 2 \theta$ por passo com tempo de contagem de $1,0 \mathrm{~s}$ por passo e no intervalo $2 \theta$ de $10^{\circ}$ a $80^{\circ}$. As curvas termogravimétricas foram obtidas em termobalança SDT Q500, TA Instruments, com taxa de aquecimento de $10{ }^{\circ} \mathrm{C} / \mathrm{min}$ até $900{ }^{\circ} \mathrm{C}$ em atmosfera de ar com vazão de $60 \mathrm{~mL} / \mathrm{min}$. Os espectros de infravermelho (FTIR) foram obtidos no espectrofotômetro IRPrestige-21, Shimadzu, sendo as amostras analisadas na forma de pastilhas de $\mathrm{KBr}$ no intervalo espectral entre 4000 e $400 \mathrm{~cm}^{-1}$ 
Tabela II - Resumo das pastas geopoliméricas produzidas. [Table II - Summary of geopolymers produced.]

\begin{tabular}{cccc}
\hline Amostra & $\begin{array}{c}\text { Razão molar } \\
\mathrm{SiO}_{2} / \mathrm{Al}_{2} \mathrm{O}_{3}\end{array}$ & $\begin{array}{c}\text { Molari- } \\
\text { dade }\end{array}$ & $\begin{array}{c}\text { Silicato } \\
\text { utilizado }\end{array}$ \\
\hline 3.0NaOH8SW & 3,0 & $8 \mathrm{M}$ & $\mathrm{SW}$ \\
3.0NaOH10SW & 3,0 & $10 \mathrm{M}$ & $\mathrm{SW}$ \\
3.5NaOH8SW & 3,5 & $8 \mathrm{M}$ & $\mathrm{SW}$ \\
3.5NaOH10SW & 3,5 & $10 \mathrm{M}$ & $\mathrm{SW}$ \\
4.0NaOH8SW & 4,0 & $8 \mathrm{M}$ & $\mathrm{SW}$ \\
4.0NaOH10SW & 4,0 & $10 \mathrm{M}$ & $\mathrm{SW}$ \\
3.0NaOH8ASW & 3,0 & $8 \mathrm{M}$ & $\mathrm{ASW}$ \\
3.0NaOH10ASW & 3,0 & $10 \mathrm{M}$ & $\mathrm{ASW}$ \\
3.5NaOH8ASW & 3,5 & $8 \mathrm{M}$ & $\mathrm{ASW}$ \\
3.5NaOH10ASW & 3,5 & $10 \mathrm{M}$ & $\mathrm{ASW}$ \\
4.0NaOH8ASW & 4,0 & $8 \mathrm{M}$ & $\mathrm{ASW}$ \\
4.0NaOH10ASW & 4,0 & $10 \mathrm{M}$ & $\mathrm{ASW}$ \\
\hline
\end{tabular}

SW: silicato de sódio comercial; ASW: silicato de sódio de CCA.

e resolução de $2 \mathrm{~cm}^{-1}$. A resistência à compressão uniaxial dos geopolímeros foi medida aos 7 e 28 dias à temperatura ambiente usando uma prensa hidráulica de acionamento elétrico e módulo eletrônico de leitura (PC200C, Imec) com capacidade de $2000 \mathrm{kN}$ e velocidade de carregamento correspondente de $0,20 \mathrm{MPa} / \mathrm{s}$. Foram ensaiadas três amostras para cada medida e determinados os valores médios.

\section{RESULTADOS E DISCUSSÃO}

Caracterização das matérias-primas e do silicato de sódio

$\mathrm{Na}$ Tabela I são apresentados os resultados de composição química. Observou-se que o metacaulim (MK) apresentou $\mathrm{SiO}_{2}$ e $\mathrm{Al}_{2} \mathrm{O}_{3}$ em elevadas proporções, 53,06\% e $36,50 \%$, respectivamente. O teor de $\mathrm{SiO}_{2}$ da cinza de casca de arroz (CCA) foi de aproximadamente $80 \%$ e os demais óxidos estavam presentes como impurezas [2]. A CCA apresentou óxido férrico, que não foi encontrado no silicato comercial. Embora a presença de ferro em geopolímeros seja um assunto ainda pouco estudado, é provável que a presença deste elemento químico altere a reação de geopolimerização [24]. Gomes et al. [25] sugerem que a relação atômica silício/alumínio seria semelhante à relação atômica silício/(alumínio+ferro) para precursores ricos em ferro. No difratograma de raios X do MK (Fig. 1a), observou-se a predominância de halo amorfo na faixa entre $15^{\circ}$ e $32^{\circ}$, indicando que houve sílica e alumina amorfa [15]; observaram-se também picos característicos de impurezas de quartzo $\left(2 \theta 26,6^{\circ}\right)$ e muscovita em menor quantidade [26, 27]. A presença dessas fases cristalinas afeta a reatividade do MK durante a geopolimerização. Pela análise do difratograma foi possível concluir que o MK não possuiu a estrutura da caulinita, caracterizando um material reativo adequado à reação de geopolimerização [13]. No difratograma de raios X da CCA (Fig. 1b), foram observados picos cristalinos característicos de cristobalita desordenada e o halo amorfo próximo de $15-30^{\circ}$. A fase cristobalita foi confirmada pelos picos em $21,94^{\circ}, 28,44^{\circ}, 30,51^{\circ}$ e $36,06^{\circ}$ [28].
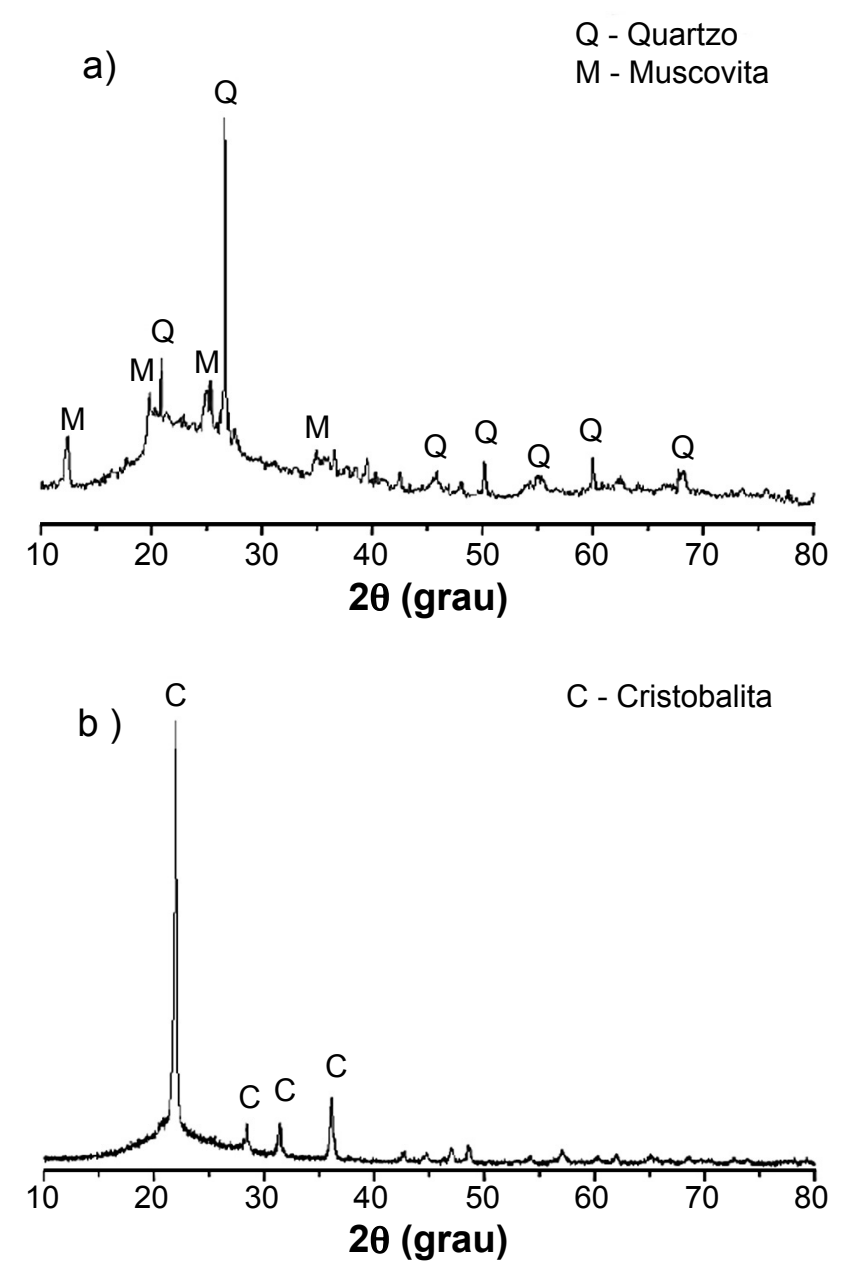

Figura 1: Difratogramas de raios $\mathrm{X}$ de: a) metacaulim (MK); e b) cinza da casca de arroz (CCA).

[Figure 1: XRD patterns of: a) metakaolin (MK); and b) rice husk $\operatorname{ash}(\mathrm{RHA})$.

A curva de termogravimetria (TG) do MK (Fig. 2a) apresentou perda de massa inicial menor que $1 \%$ relacionada à umidade. No intervalo entre 400 e $800{ }^{\circ} \mathrm{C}$, a perda de massa foi da ordem de $8 \%$ atribuída à liberação das hidroxilas estruturais, sugerindo que o processo industrial foi eficiente e que a amostra foi predominantemente MK [29], conforme já sugerido pelos dados de DRX (Fig. 1a). Em relação à CCA (Fig. 2b), a análise da decomposição térmica indicou perda de aproximadamente 3\% de massa até 100 ${ }^{\circ} \mathrm{C}$. Essa região foi relacionada à perda de água fisicamente adsorvida. Entre 102 e $600{ }^{\circ} \mathrm{C}$, observou-se a decomposição da celulose e da lignina [22]. Na curva termogravimétrica da CCA, houve um conjunto de eventos sucessivos entre $350 \mathrm{e}$ $900{ }^{\circ} \mathrm{C}$ totalizando aproximadamente $8 \%$ de perda de massa. 
Não foi possível distinguir os processos individuais nas condições que foi conduzido o experimento, mas as etapas previstas até $600{ }^{\circ} \mathrm{C}$ devem ter se somado a outros eventos de pirólise da matéria orgânica remanescente. De qualquer forma, menos de $10 \%$ da amostra foi material orgânico, o que mostrou que esta cinza teve um adequado tratamento térmico [30].
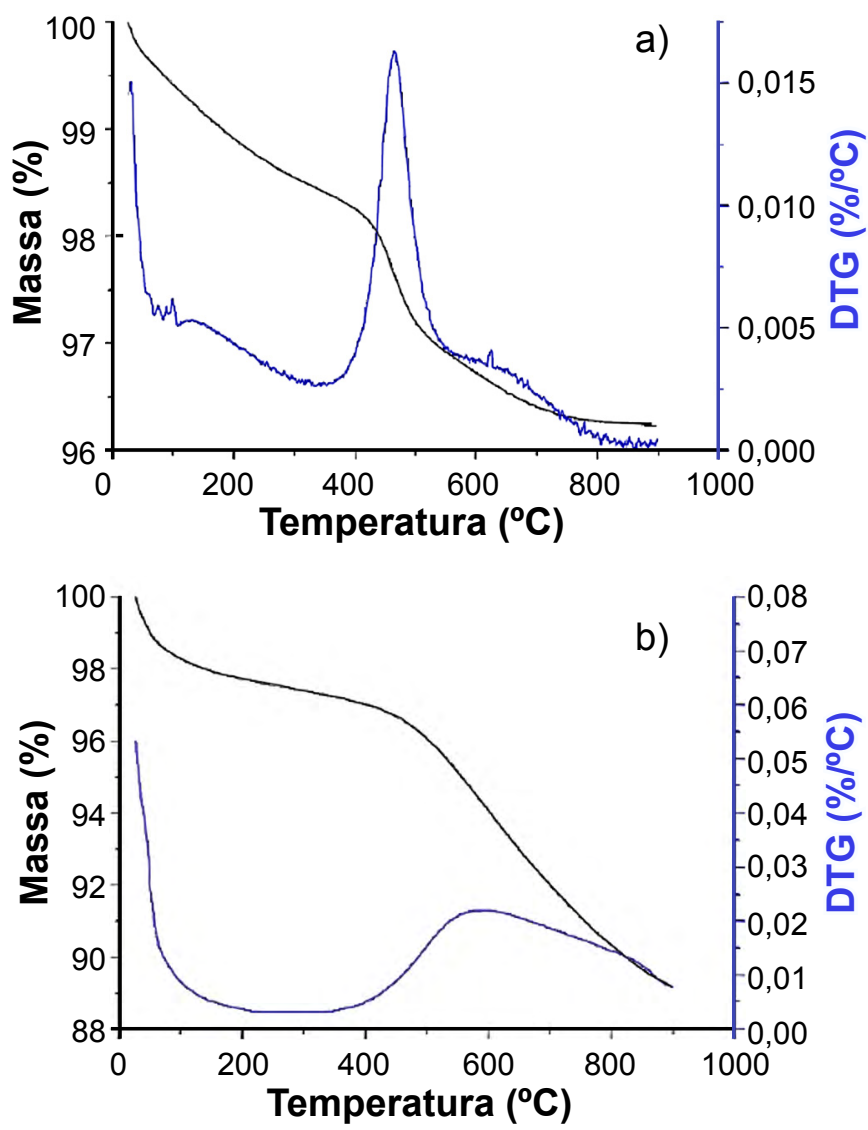

Figura 2: Curvas de TG e DTG (derivada da TG) de: a) MK; e b) CCA.

[Figure 2: TG and DTG (derivative of TG) curves of: a) MK; and b) RHA.]

$\mathrm{O}$ estiramento assimétrico da ligação $\mathrm{Si}-\mathrm{O}-\mathrm{Si}$ do MK (Fig. 3a) aconteceu no intervalo entre $1074 \mathrm{e} 811 \mathrm{~cm}^{-1}$ devido à presença de sílica amorfa [26]. No espectro FTIR mostrado na Fig. 3a, observa-se um ombro em $1069 \mathrm{~cm}^{-1}$ atribuído a este estiramento. As bandas características da caulinita entre 1400 a $400 \mathrm{~cm}^{-1}$ não foram observadas no espectro do MK. As bandas em 777, 1345 e $469 \mathrm{~cm}^{-1}$ são características do MK. De acordo com esses dados, o material passou por um eficiente processo de desidroxilação, com potencial de ser um bom precursor geopolimérico. No espectro FTIR da CCA (Fig. 3b), as vibrações em $3450 \mathrm{~cm}^{-1}$ foram características do estiramento -OH, $\mathrm{HOH}$ [31-34]. O pico em $1090 \mathrm{~cm}^{-1}$ foi relacionado à vibração de alongamento assimétrico $\mathrm{Si}-\mathrm{O}-\mathrm{Si}$ e dá uma indicação do comprimento e ângulo das ligações em uma rede de silicato [2]. O pico observado em $798 \mathrm{~cm}^{-1}$ correspondeu à vibração de alongamento simétrico Si-O-
Si e o pico em $465 \mathrm{~cm}^{-1}$ foram de vibrações de deformação angular Si-O-Si ou O-Si-O. Identificou-se também um pico em $465 \mathrm{~cm}^{-1}$ associado ao grau de 'amorfização' do material [28].
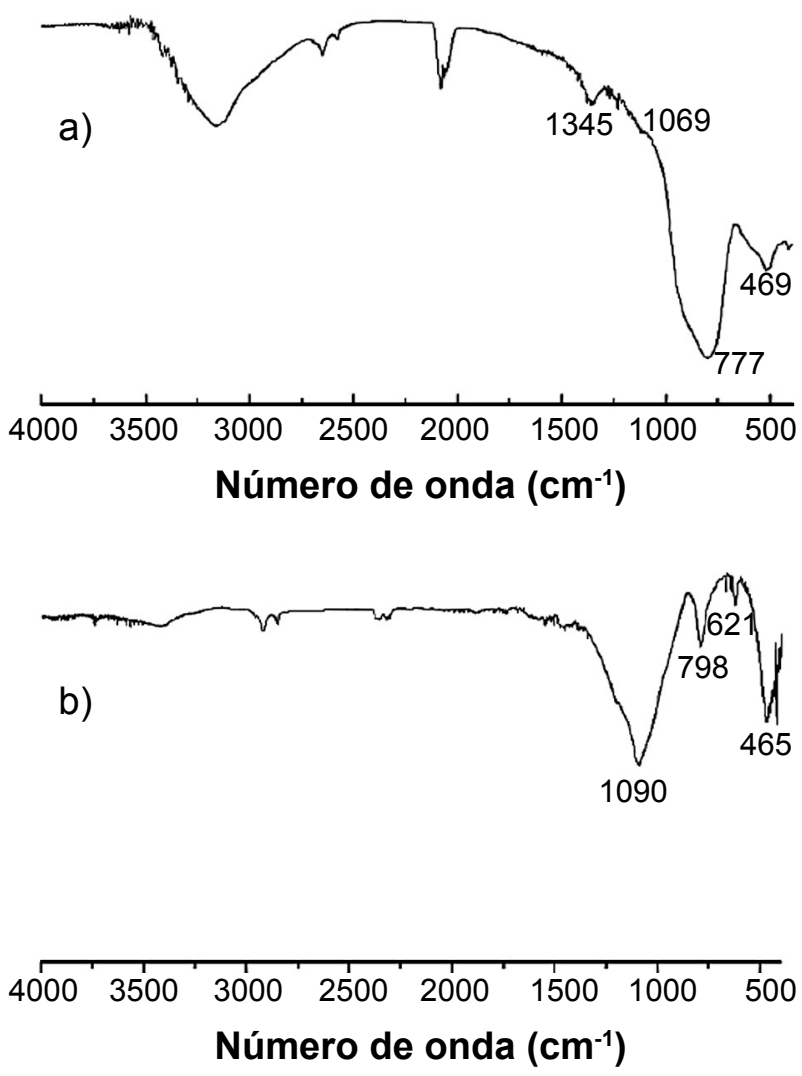

Figura 3: Espectros de FTIR de: a) MK; e b) CCA. [Figure 3: FTIR spectra of: a) MK; and b) RHA.]

\section{Caracterização dos geopolímeros}

Resistência à compressão: na Fig. 4a são apresentados os dados de resistência à compressão dos geopolímeros produzidos a partir do silicato de sódio comercial, onde se pode verificar que a relação $\mathrm{SiO}_{2} / \mathrm{Al}_{2} \mathrm{O}_{3}$ igual a 4,0 resultou no melhor desempenho, com incremento aos 28 dias. Para geopolímeros, o aumento da resistência à compressão está associado à maior dissolução dos aluminossilicatos, que são determinantes no processo de formaçãodarede tridimensional [35]. Os dados de resistência à compressão dos geopolímeros produzidos com silicato de sódio de cinza de casca de arroz (ASW) estão apresentados na Fig. 4b. Observou-se que a maior resistência à compressão também foi da amostra com razão $\mathrm{SiO} / \mathrm{Al}_{2} \mathrm{O}_{3}$ de 4,0 e concentração de hidróxido de sódio igual a $10 \mathrm{M}$, podendo estar associada com a formação de oligômeros [36]. Observam-se resultados semelhantes na literatura $[2,6,35]$, confirmando que o silicato de sódio produzido com a cinza da casa de arroz contribuiu para formação de estrutura mais densa e compacta, aumentando a resistência do geopolímero. A resistência à compressão mais baixa foi relacionada com a menor quantidade de átomos de Si e razoável ausência de sílica solúvel do silicato de sódio 

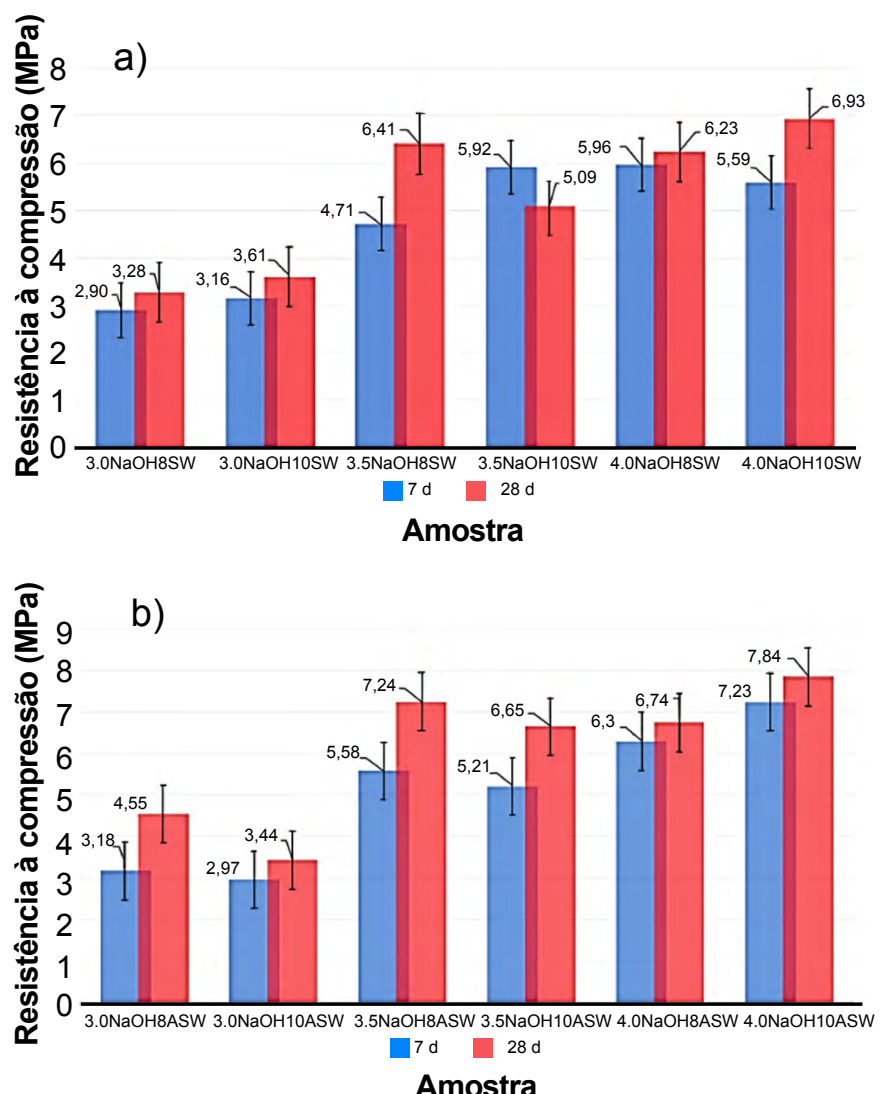

Figura 4: Resistência à compressão dos geopolímeros fabricados com SW (a) e ASW (b).

[Figure 4: Compressive strength of geopolymers prepared with SW (a) and $A S W$ (b).]

a partir da cinza da casca de arroz [2]. O excesso de álcali propicia a formação de carbonato de sódio, logo, os álcalis devem ser suficientes apenas para garantir o equilíbrio das redes de geopolimerização.

Espectroscopia no infravermelho: nos espectros FTIR dos geopolímeros (Fig. 5) foi identificado que ocorreu o processo de geopolimerização através da formação de um gel, comprovado pelas bandas entre 1001 e $1024 \mathrm{~cm}^{-1}$. Podese considerar o grau de geopolimerização através destas bandas características, que são atribuídas ao estiramento assimétrico das ligações Si-O-Si e Si-O-Al [2]. Observouse que os picos em 1069 e $798 \mathrm{~cm}^{-1}$ nos espectros de infravermelho do MK e da CCA, respectivamente, sofreram deslocamento no processo de geopolimerização para o número de onda a $1001 \mathrm{~cm}^{-1}$ proveniente da dissolução dos materiais, gerando a reorganização das redes de aluminossilicatos [37]. A presença de bandas próximas a $1567 \mathrm{~cm}^{-1}$ atribuídas a carbonatos pode ser explicada pela existência de hidróxido de sódio ou silicato de sódio que não participaram da reação de geopolimerização, deixando íons $\mathrm{Na}^{+}$disponíveis para reagir com $\mathrm{CO}_{2}$ atmosférico [37]. Em geopolímeros, a frequência das bandas de absorção está relacionada com a razão $\mathrm{Si} / \mathrm{Al}$; quanto maior a quantidade de átomos de alumínio, maior é a tendência de deslocamento dos picos de absorção de cerca de $80-90 \mathrm{~cm}^{-1}$ na direção dos números de onda mais baixos [2, 37].
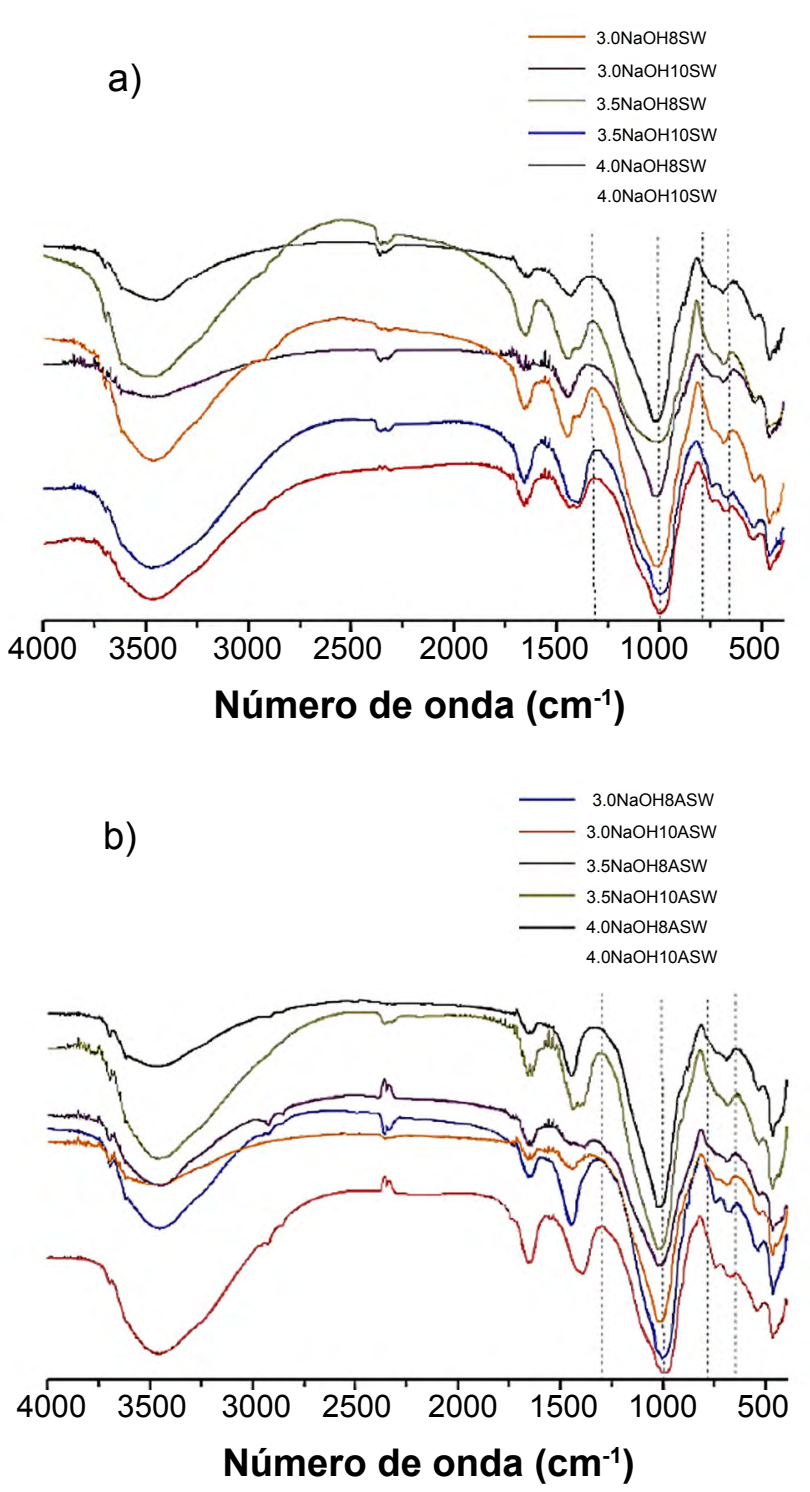

Figura 5: Espectro de FTIR de geopolímeros fabricados com SW (a) e ASW (b).

[Figure 5: FTIR spectra of geopolymers prepared with SW (a) and $A S W(b)$.

Os espectros FTIR das amostras de geopolímeros produzidas com o silicato de sódio de cinza de casca de arroz (ASW) mostraram a dissolução da cinza da casca de arroz através da ausência do pico característico em 850 $\mathrm{cm}^{-1}$ e formação de bandas entre 706 e $714 \mathrm{~cm}^{-1}$ referentes aos tetraedros dos geopolímeros [17]. Nos espectros foi observado pico em $710 \mathrm{~cm}^{-1}$, sugerindo a formação de $\mathrm{Al}$ tetraédrico relacionada ao processo de geopolimerização [37]. $\mathrm{O}$ pico em $501 \mathrm{~cm}^{-1}$ se refere à formação de material cristalino através da ligação $\mathrm{Si}-\mathrm{O}-\mathrm{Si}$, sugerindo que as fases encontradas nos precursores geopoliméricos não participaram da reação, comprovando a atribuição de sílica cristalina [2,37]. As bandas próximas à região de $460 \mathrm{~cm}^{-1}$ são relacionadas aos planos de curvatura de $\mathrm{Al}-\mathrm{O}$ e Si-O que são encontrados na faixa de 463 a $672 \mathrm{~cm}^{-1}$ [37]. As bandas entre 950 e $1150 \mathrm{~cm}^{-1}$ resultaram da reação de geopolimerização do 


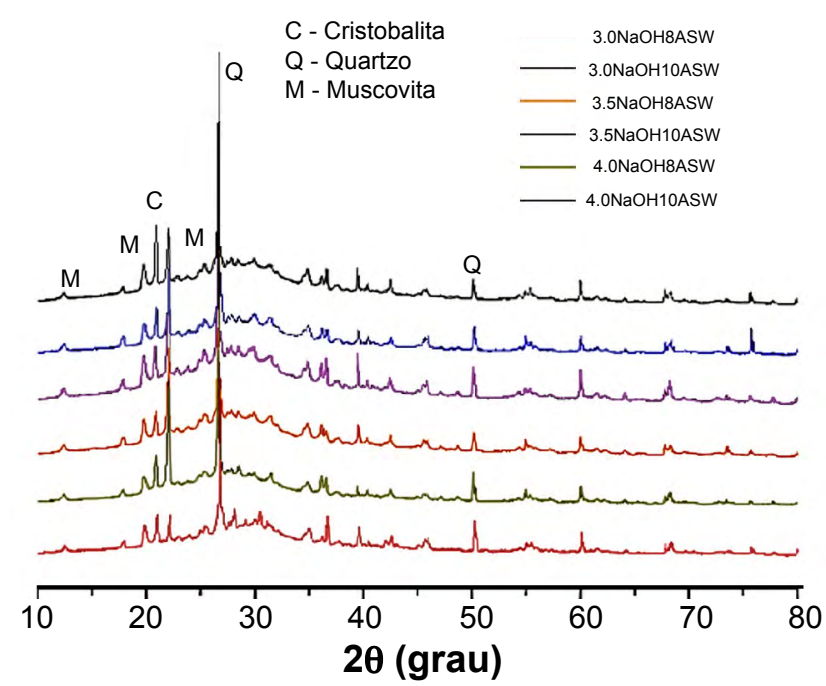

Figura 6: Difratogramas de raios $\mathrm{X}$ dos geopolímeros produzidos com silicato de sódio de CCA (ASW).

[Figure 6: X-ray diffraction patterns of geopolymers prepared with ASW.]

metacaulim com a solução ativadora [10]. Todas as amostras de geopolímeros produzidas com silicato de sódio comercial (SW) e silicato de sódio de cinza de casca de arroz (ASW) apresentaram bandas entre 998 e $1038 \mathrm{~cm}^{-1}$ com estiramento assimétrico de $\mathrm{Si}-\mathrm{O}-\mathrm{Si}$ e $\mathrm{Si}-\mathrm{O}-\mathrm{Al}$, sugerindo que em todas as amostras ocorreu a formação de geopolímero.

Difratogramas de raios $X$ de geopolímeros com silicato de sódio de CCA ( $A S W)$ : na Fig. 6 são mostrados os difratogramas de raios $\mathrm{X}$ dos geopolímeros produzidos com silicato de sódio de cinza de casca de arroz. Foi possível identificar uma predominância de um halo amorfo característico em todas as amostras no intervalo $2 \theta$ de $20^{\circ}$ a $35^{\circ}$ [12], indicando a formação de um gel amorfo durante o processo de geopolimerização. $\mathrm{O}$ quartzo e a muscovita foram as principais fases cristalinas encontradas nos geopolímeros produzidos e foram oriundas do metacaulim utilizado na reação. Isso sugeriu que estas formas de sílica não reagem. Entretanto, observou-se que a cristobalita, forma de sílica mais reativa, parece participar da reação, uma vez que a intensidade dos picos desta fase foi reduzida nos geopolímeros.

\section{CONCLUSÕES}

De acordo com os dados obtidos, foi possível usar cinza de casca de arroz (CCA) para produzir geopolímeros. Os resultados sugeriram que tanto a concentração de $\mathrm{NaOH}$ quanto a relação $\mathrm{SiO}_{2} / \mathrm{Al}_{2} \mathrm{O}_{3}$ afetam muito a resistência à compressão das amostras de geopolímeros resultantes. Acredita-se que as impurezas presentes na CCA não afetaram o sistema, uma vez que os melhores resultados foram alcançados nas amostras com maior razão molar $\mathrm{SiO}_{2} / \mathrm{Al}_{2} \mathrm{O}_{3}$, portanto, com maior quantidade de $\mathrm{CCA}$. As amostras de geopolímero preparadas com solução de $\mathrm{NaOH} 10 \mathrm{M}$ e silicato de sódio com cinza de casca de arroz apresentaram resistência à compressão que foram comparáveis ou mesmo superiores às amostras produzidas com silicato de sódio comercial. Os dados de FTIR confirmaram que a geopolimerização ocorreu de maneira similar nas amostras independente do silicato de sódio utilizado.

\section{AGRADECIMENTOS}

Os autores agradecem ao $\mathrm{CNPq}$ pelo apoio financeiro Projeto Casadinho/PROCAD, e às empresas Metacaulim do Brasil e Diatom Mineração pelas amostras doadas.

\section{REFERÊNCIAS}

[1] M. Xu, Y. He, C. Wang, X. He, X. He, J. Liu, X. Cui, Appl. Clay Sci. 115 (2015) 254.

[2] H.K. Tchakouté, C.H. Rüscher, S. Kong, E. Kamseu, C. Leonelli, Constr. Build. Mater. 114 (2016) 276.

[3] E. Najafi Kani, A. Allahverdi, J.L. Provis, Cem. Concr. Compos. 34 (2012) 25.

[4] R. Pouhet, M. Cyr, Constr. Build. Mater. 120 (2016) 150.

[5] P. Duxson, S.W. Mallicoat, G.C. Lukey, W.M. Kriven, J.S.J. van Deventer, Colloid Surface A 292 (2007) 8.

[6] H.K. Tchakoute, C.H. Rüscher, J.N.Y. Djobo, B.B.D. Kenne, D. Njopwouo, Appl. Clay Sci. 107 (2015) 188.

[7] S. Zeng, J. Wang, Constr. Build. Mater. 121 (2016) 386.

[8] T. Wang, Y. Xue, M. Zhou, Y. Lv, Y. Chen, S. Wu, H. Hou, Constr. Build. Mater. 131 (2017) 361.

[9] B.C. McLellan, R.P. Williams, J. Lay, A. van Riessen, G.D. Corder, J. Clean. Prod. 19 (2011) 1080.

[10] M. Criado, A. Palomo, A. Fernandez-Jimenez, Fuel 84 (2005) 2048.

[11] K.-H. Yang, J.-K. Song, A.F. Ashour, E.-T. Lee, Constr. Build. Mater. 22 (2008) 1981.

[12] M.A. Villaquirán-Caicedo, R.M. de Gutiérrez, Mater. Lett. 230 (2018) 300.

[13] R. Pode, Renew. Sust. Energ. Rev. 53 (2016) 1468.

[14] K.K. Rao, P. Pranav, D. Anusha, Int. J. Eng. Sci. Technol. 3 (2011) 8076.

[15] J. He, "Synthesis and characterization of geopolymers for infrastructural applications", Doct. Diss., LSU, Louisiana (2012).

[16] U. Kalapathy, Bioresour. Technol. 73 (2000) 257.

[17] E.L. Foletto, E. Gratieri, L.H. de Oliveira, S.L. Jahn, Mater. Res. 9 (2006) 335.

[18] V. Živica, Cem. Concr. Compos. 28 (2006) 21.

[19] S.A. Bernal, E.D. Rodríguez, R. Mejia De Gutiérrez, J.L. Provis, S. Delvasto, Waste Biomass Valori. 3 (2012) 99. [20] E. Kamseu, L.M. Beleuk à Moungam, M. Cannio, N. Billong, D. Chaysuwan, U.C. Melo, C. Leonelli, J. Clean. Prod. 142 (2017) 3050.

[21] FAO, "The state of food and agriculture: innovation in family farming”, Food Agric. Org. UN, Rome (2017).

[22] D. Geetha, A. Ananthiand, P.S. Ramesh, Res. Rev. J. Pure Appl. Phys. 4 (2016) 20.

[23] F. Slaty, H. Khoury, H. Rahier, J. Wastiels, Appl. Clay Sci. 104 (2015) 229. 
[24] J.S. Lima, "Efeito da relação molar $\mathrm{SiO}_{2} / \mathrm{Al}_{2} \mathrm{O}_{3}$ e das condições de cura nas propriedades de geopolímeros obtidos com silicato de cinza da casca de arroz", Diss. Mestr., Un. Fed. Pernambuco, Caruaru (2018).

[25] K.C. Gomes, S.M. Torres, Z.E. da Silva, N. Perazzo Barbosa, M.R.F. Lima Filho, Key Eng. Mater. 600 (2014) 329.

[26] M. Sarkar, K. Dana, S. Das, J. Mol. Struct. 1098 (2015) 110.

[27] I. Ozer, S. Soyer-Uzun, Ceram. Int. 41 (2015) 10192.

[28] C.L. Hwang, T.P. Huynh, Constr. Build. Mater. 101 (2015) 1.

[29] A. Gharzouni, E. Joussein, B. Samet, S. Baklouti, S. Rossignol, J. Non Cryst. Solids 410 (2015) 127.

[30] G.C. Cordeiro, R.D. Toledo Filho, E.M.R. Fairbairn,
Amb. Constr. 9 (2009) 99.

[31] P. Yu, R.J. Kirkpatrick, B. Poe, P.F. McMillan, X. Cong, J. Am. Ceram. Soc. 82 (2004) 742.

[32] R. García, R. Vigil de la Villa, I. Vegas, M. Frías, M.I. Sánchez de Rojas, Constr. Build. Mater. 22 (2008) 1484.

[33] I. García Lodeiro, A. Fernández-Jimenez, A. Palomo, D. Macphee, Cem. Concr. Res. 40 (2010) 27.

[34] J. Madejova, P. Komadel, Clays Clay Miner. 49 (2001) 410.

[35] Y.M. Liew, C.Y. Heah, A.B. Mohd Mustafa, H. Kamarudin, Prog. Mater. Sci. 83 (2016) 595.

[36] H. Xu, J.S.J. van Deventer, Miner. Eng. 15 (2002) 1131. [37] M. Romagnoli, C. Leonelli, E. Kamse, M. Lassinantti Gualtieri, Constr. Build. Mater. 36 (2012) 251.

(Rec. 05/07/2019, Rev. 29/12/2019, 04/01/2020, Ac. $07 / 01 / 2020)$ 